\title{
El modelo de los cinco grandes factores de personalidad y el uso problemático de Internet en jóvenes colombianos
}

\section{The model of the big five personality factors and problematic Internet use in Colombian youth}

\author{
Diana Ximena Puerta-Cortés*, **; Xavier Carbonell** \\ * Universidad de Ibagué. Tolima, Colombia; ** Universitat Ramón Llull. FPCEE. Barcelona, España.
}

\section{Resumen}

El objetivo del estudio fue relacionar las dimensiones básicas de personalidad formuladas por el modelo de los cinco grandes factores con el uso problemático de Internet, en una muestra de 411 jóvenes colombianos de 18 a 28 años de tres universidades privadas. Se les administraron online: el cuestionario de datos socio-demográficos y hábitos de uso de Internet, el Big Five Inventory (John, Donahue y Kentle, 1991), para evaluar la personalidad y el Internet Addiction Test (Young, 1998), para determinar el grado de uso de Internet (controlado, problemático o adictivo). Los resultados revelaron que el $9.7 \%$ de la muestra presenta un uso problemático de Internet. Este porcentaje era en su mayoría masculino $\left(\chi^{2}=12.93 ; p=0.01\right)$ y realizaba actividades de comunicación y ocio. El uso problemático correlaciona positivamente con neuroticismo y negativamente con afabilidad y responsabilidad. Por otra parte, no tiene relación con extraversión y apertura a la experiencia. Ser mujer y la dimensión de responsabilidad son factores protectores del uso problemático, mientras que el neuroticismo lo predice. En conclusión, los datos aportan evidencia empírica en el estudio de la relación entre la personalidad y el uso problemático de Internet.

Palabras clave: uso de Internet, adicción a Internet, modelo de personalidad de los cinco factores, estudiantes universitarios, Colombia.

\begin{abstract}
The aim of the study was to relate the basic dimensions of personality formulated by the model of the big five factors with problematic Internet use in a sample of 411 Colombian youngsters, 18-28 years of age, attending three private universities. Online survey questionnaires were administered for: socio-demographics and Internet usage habits, the Big Five Inventory (John, Donahue and Kentle, 1991), to assess personality, and the Internet Addiction Test (Young, 1998), to determine the degree of use of the Internet (controlled, problematic or addictive). The results revealed that $9.7 \%$ of the sample has a problematic Internet use. Among them, the majority were male $(\chi 2=$ 12.93, $\mathrm{p}=0.01$ ) and performing communication and leisure activities. The problematic use correlates positively with neuroticism and negatively with friendliness and responsibility. On the other hand, is not related to extraversion and openness to experience. Being female and the responsibility dimension are protective factors from problematic use, while neuroticism predicts it. In conclusion, the study data provides empirical evidence of the relationship between personality and problematic Internet use.

Key words: Internet usage, Internet addiction, five factors personality model, college students, Colombia.
\end{abstract}


$\mathrm{N}$ avegar por Internet se ha convertido en una de las actividades preferentes para los jóvenes, debido al fácil acceso libre e ilimitado, la rapidez en la respuesta, la interactividad y las recompensas inmediatas (Echeburúa y De Corral, 2010; Young, 2004). A pesar de las múltiples ventajas de esta tecnología, algunos usuarios hacen un uso problemático (Caplan, 2003) o adictivo (Young, 1998; Sánchez-Carbonell, Beranuy, Castellana, Chamarro y Oberst, 2008). El uso problemático de Internet se caracteriza por el descuido de actividades académicas, laborales o domésticas y su sustitución por actividades en línea, como el correo electrónico, las redes sociales, el juego y la pornografía (Caplan, 2003; Echeburúa et al., 2010; Yellowlees y Marcas, 2007).

El uso que hacen los jóvenes de Internet ha suscitado un gran interés a la hora de detectar la prevalencia de su uso problemático. Los jóvenes son un grupo vulnerable debido a las características psicológicas propias de su momento evolutivo y a sus condiciones vitales, muchos de ellos se alejan de la familia y círculo social para seguir sus estudios y se enfrentan a nuevos retos profesionales (Young, 2004).

La prevalencia juvenil de uso problemático de Internet en Europa oscila entre el $4.2 \%$ y el $6.1 \%$ (Carbonell et al., 2012; Kuss, Griffist y Binder, 2013; van den Eijnden, Spijkeman, Vermulst, van Rooij y Engels, 2009). Cabe destacar el proyecto Seyle, con 11.956 participantes de once países europeos, en el que la prevalencia fue del $4.4 \%$, mayor en hombres que en mujeres (Durke et al., 2012). En Estados Unidos, Moreno, Jelenchick, Cox, Young y Christakis (2011), a partir de una revisión de dieciocho estudios, identificaron una prevalencia entre el $0 \%$ y el $26.3 \%$ y en Asia, entre el $1.6 \%$ y el $18.4 \%$ (Cao y Su, 2007; Kim et al., 2006; Liu et al., 2009). Según la literatura no se puede establecer con claridad si la variabilidad en los resultados de prevalencia se relaciona con las diferencias culturales entre regiones o con los diversos enfoques en la definición de uso problemático de Internet (Moreno et al., 2011).

La psicología de la personalidad se ha interesado por estudiar la relación entre las diferencias individuales y el uso de Internet. La población de usuarios de Internet no es uniforme y utiliza la red en consonancia con su preferencia personal y su personalidad (Amichai-Hamburger, 2002). Para Montag, Jurkiewicz y Reuter (2010), la personalidad es un factor importante para examinar la predisposición al uso problemático de Internet.

Uno de los modelos de personalidad más aceptados es el de los cinco grandes factores de McCrae y Costa (2008). Su historia se inició en la década de los cuarenta, pero fue en los años ochenta cuando se desarrolló el modelo que actualmente se conoce. Surgió a partir de una variedad de factores analíticos, léxicos y de temperamento realizados con escalas de personalidad (Goldberg, 1993). Se comprobó además, su consistencia y posibilidad de generalizarlo a diversos contexos (Fossati, Borroni, Marchione y Maffei, 2011; Triandis y Eunkook, 2002). McCrae et al. (1997) desarrollaron el Inventario de Personalidad NEO PI de Cinco Factores y, para responder a las necesidades de un cuestionario corto, John, Donahue y Kentle (1991) construyeron el Big Five Inventory (BFI). Los cinco factores relativamente independientes que dan cuenta de la variación fenotípica de la personalidad son extraversión, afabilidad, responsabilidad, neuroticismo y apertura a la experiencia (John y Srivastava, 1999; Thalmayer, Saucier y Eigenhuis, 2011).

La relación entre las dimensiones de personalidad del modelo de los cinco grandes factores y el uso problemático de Internet ha sido explorada por varios autores. Algunos de los estudios reportan una relación positiva con las dimensiones de neuroticismo (Hamburger y Ben-Artzi, 2000; Hardie y Tee, 2007; Tsai et al., 2009; Wolfradt y Doll, 2001) y extraversión (Hamburger et al., 2000). Otros muestran relación negativa con neuroticismo y positiva con apertura y afabilidad (Luo y Ding, 2006). Y otros, puntuaciones elevadas en neuroticismo y bajas en extraversión (Viñas, 2009). Para Landers y Lounsbury (2006) los extrovertidos pasan menos tiempo en línea a diferencia de los introvertidos, quienes prefieren comunicarse virtualmente y evitar la interacción cara a cara. Buckner, Castille y Sheets (2012) establecen que los resultados de la dimensión apertura a la experiencia son inconsistentes. Engelberg y Sjöberg (2004) y Witt, Massman y Jackson (2011) concluyeron que las relaciones entre las dimensiones de personalidad del modelo de los cinco grandes factores y el uso de Internet no son evidentes.

Los estudios mencionados muestran resultados interesantes, pero es insuficiente la relación entre sus hallazgos, por lo que se dispone de poca información para guiar las predicciones sobre la base del modelo de los cinco grandes factores y el uso problemático de Internet. Según Buckner et al. (2012), la mayoría de estudios solo han examinado las relaciones entre el uso de Internet y las dimensiones de neuroticismo y extraversión. En este sentido, es necesario realizar investigaciones, para conocer la personalidad, los hábitos y grado de uso en muestras de países en desarrollo, donde el grado de penetración de Internet es superior (39\%) a la media mundial $(33 \%)$ (Tendencias Digitales, 2012). El conocimiento del perfil de los jóvenes con uso problemático de Internet, puede ayudar a detectar los usuarios en riesgo y evitar el uso inadecuado de esta tecnología.

En consecuencia, éste estudio exploratorio se diseñó con el objetivo de relacionar las dimensiones básicas de personalidad formuladas por el modelo de los cinco grandes factores con el uso problemático de Internet en jóvenes colombianos. 


\section{Método}

\section{Participantes}

En el estudio participaron 411 jóvenes de tres universidades privadas de Colombia. Los estudiantes fueron reclutados a través de la página web de las universidades, redes sociales, blogs y correos electrónicos. Los participantes fueron seleccionados mediante un procedimiento de muestreo intencional, mediante la inclusión de los estudiantes que respondieron a la invitación para el estudio. De ellos, el $61.3 \%$ fueron mujeres y el $38.7 \%$ hombres; el $87.3 \%$ eran estudiantes de grado y el $12.6 \%$ de postgrado. Su edad media fue de 20.80 años ( $D T=3.77$; rango 18-28), 20.62 años las mujeres $(D T=3.23)$ y 21.08 los hombres $(D T=4.48)$. En cuanto al estado civil, el $91.8 \%$ estaba soltero y $8.1 \%$ casado. La participación en el estudio fue voluntaria y se garantizó la confidencialidad y el anonimato de sus respuestas.

\section{Instrumentos}

Cuestionario de datos socio-demográficos y hábitos de uso de Internet. Cuestionario de elaboración propia que consta de dos apartados. El primero refiere a la información de la edad, el sexo, la ciudad, el estado civil y el nivel de estudios. El segundo a los hábitos de uso de Internet (lugar de acceso, horas diarias de uso, franja horaria) y las actividades que realizan en la red: comunicación (redes sociales, chat, juegos); información (académica, radio por Internet, periódicos) y ocio (subastas, videos, contenido para adultos, navegar en la red).

Test de Adicción a Internet (IAT). El IAT (Young, 1998) es un instrumento de 20 ítems que evalúa el impacto del uso de Internet en las interacciones sociales y la influencia del uso de Internet en la vida diaria. Este test permite categorizar las respuestas de los usuarios de Internet en tres grupos: uso controlado (de 20 a 49 puntos), uso problemático (de 50 a 79 puntos) y uso adictivo (de 80 a 100 puntos). Fue traducido al castellano por Estévez, Bayón, de la Cruz y Fernández (2009). Adaptado y validado a la población colombiana por Puerta-Cortés, Carbonell y Chamarro (2012) y Puerta-Cortés y Carbonell (2013), con una fiabilidad buena. La fiabilidad de la aplicación online del IAT para este estudio fue de $\alpha=0.84$.

Inventario de Personalidad de los cinco factores (BFI). El Big Five Inventory (John et al., 1991) evalúa las cinco grandes dimensiones de personalidad (extraversión, afabilidad, responsabilidad, neuroticismo y apertura). Está compuesto por 44 ítems tipo Likert de cinco opciones de respuesta, que van desde el total acuerdo hasta el total desacuerdo. Para nuestro estudio se utilizó la versión adaptada a contextos de habla hispana por Benet-Martínez y Jhon (1988). El análisis de fiabilidad $\alpha$ de Cronbach del BFI fue de $=0.73 \mathrm{y}$, para cada uno de los factores correspondientes a extraversión
( $\alpha=0.67)$, afabilidad $(\alpha=0.66)$, responsabilidad $(\alpha=0.68)$, neuroticismo $(\alpha=0.63)$ y apertura $(\alpha=0.60)$.

\section{Procedimiento}

A partir de la inscripción online, los participantes recibieron en sus correos electrónicos la declaración de consentimiento informado, el cuestionario de datos socio-demográficos y hábitos de uso de Internet, el IAT y el BFI. Los cuestionarios se adaptaron para la aplicación online al software SurveyMonkey ${ }^{\circledR}$ disponible en (http:// es.surveymonkey.com/), proveedor de encuestas online, que ofrece seguridad SSL para la autenticación y privacidad de la información y exporta los resultados de los cuestionarios al programa Excel. El enlace obtenido del cuestionario fue copiado en el muro de la red social Facebook de los asistentes de investigación y en los correos electrónicos de los participantes inscritos.

\section{Análisis de datos}

Con el paquete estadístico SPSS 19, se realizaron análisis bivariados aplicando el test $\chi^{2}$ para detectar y describir la relación entre las variables socio-demográficas, las actividades y categorías de uso de Internet. El coeficiente de Pearson se utilizó para analizar la correlación lineal entre cada una de las dimensiones del modelo de personalidad y las puntuaciones medias del IAT. Se valoró la fiabilidad de los cuestionarios mediante el índice de consistencia interna Alfa de Cronbach, calculado con el método de la varianza de los ítems y se hizo un análisis de regresión logística binaria para detectar las variables predictivas del uso problemático de Internet.

\section{Resultados}

\section{Prevalencia}

El 90.2\% ( $\mathrm{n}=371)$ de los participantes en el estudio tenía un uso controlado de Internet, el 9.7\% ( $n=40)$ un uso problemático y ninguno presentó un uso adictivo. En la tabla 1 se muestran las categorías de uso de Internet y su relación con el sexo, las horas de conexión y el lugar de acceso. Se observaron diferencias estadísticamente significativas entre el sexo y las categorías de uso de Internet; los hombres presentaron mayor uso problemático que las mujeres $\left(\chi^{2}=\right.$ $12.93 ; p=.01)$. La duración media de la conexión fue de 3.63 horas $(D T=2.9)$; el grupo con uso problemático permaneció en Internet más tiempo $(M=4.93 \mathrm{~h} ; D T=4.16)$ que el grupo con uso controlado $(M=3.48$ horas, $D T=2.71)$. Los participantes accedían a Internet preferentemente desde el ordenador doméstico (72\%) y el móvil $(21.7 \%)$, por la noche $(60.6 \%)$ y por la tarde $(22.4 \%)$; no se encontraron diferencias entre la franja horaria de uso de Internet y las categorías de uso controlado y problemático. 
Tabla 1

Hábitos de uso de Internet en los grupos: controlado y problemático

\begin{tabular}{|c|c|c|c|c|c|c|c|c|}
\hline \multirow{2}{*}{ Variable } & \multicolumn{2}{|c|}{ Controlado } & \multicolumn{2}{|c|}{ Problemático } & \multirow{2}{*}{ Total } & \multirow{2}{*}{$\%$ total } & \multirow{2}{*}{$\chi^{2}$} & \multirow{2}{*}{$p$} \\
\hline & $\mathrm{n}$ & $\%$ & $\mathbf{n}$ & $\%$ & & & & \\
\hline \multicolumn{9}{|l|}{ Sexo } \\
\hline Hombre & 133 & 35.8 & 26 & 65 & 159 & 38.7 & \multirow{2}{*}{12.93} & \multirow{2}{*}{.01} \\
\hline Mujer & 238 & 62.2 & 14 & 35 & 252 & 61.3 & & \\
\hline \multicolumn{9}{|l|}{ Acceso Internet } \\
\hline Ordenador doméstico & 231 & 62.2 & 30 & 75 & 296 & 72 & 2.80 & .09 \\
\hline Móvil & 70 & 18.8 & 5 & 12.5 & 89 & 21.7 & .83 & .36 \\
\hline Universidad & 60 & 16.1 & 5 & 12.5 & 15 & 3.6 & .98 & .32 \\
\hline Cibercafé & 10 & 2.6 & 0 & 0 & 11 & 2.6 & 1.21 & .27 \\
\hline \multicolumn{9}{|l|}{ Franja horaria } \\
\hline Mañana & 25 & 6.7 & 3 & 7.5 & 28 & 6.8 & .13 & .90 \\
\hline Tarde & 82 & 22.1 & 10 & 25 & 92 & 22.4 & .24 & .88 \\
\hline Noche & 188 & 50.8 & 20 & 50 & 208 & 50.6 & .10 & .74 \\
\hline Madrugada & 6 & 1.6 & 0 & 0 & 6 & 1.5 & .65 & .41 \\
\hline $24 \mathrm{hrs}$ & 70 & 18.8 & 7 & 17.5 & 77 & 18.7 & .06 & .79 \\
\hline \multicolumn{9}{|l|}{ Hrs uso diario } \\
\hline 1 a 3 & 221 & 59.6 & 19 & 47.5 & 240 & 58.3 & \multirow{5}{*}{20.20} & \multirow{5}{*}{.01} \\
\hline 4 a 6 & 119 & 32 & 10 & 25 & 129 & 31.3 & & \\
\hline 7 a 9 & 18 & 4.8 & 6 & 15 & 24 & 5.8 & & \\
\hline 10 a 12 & 5 & 1.4 & 4 & 10 & 9 & 2.2 & & \\
\hline$\rightarrow 12$ & 8 & 2.1 & 1 & 2.5 & 9 & 2.2 & & \\
\hline
\end{tabular}

Diferencias entre las categorías de uso, las actividades en Internet y el sexo

La tabla 2 muestra las actividades que realizaban en Internet los grupos con uso controlado y problemático. Los jóvenes que hacían un uso problemático de Internet accedían más a las actividades de comunicación como el correo electrónico $\left(\chi^{2}=11.61 ; p=.04\right)$ y las redes sociales $\left(\chi^{2}=14.65\right.$; $p=.01)$, actividades de ocio como las subastas $\left(\chi^{2}=14.81 ; p=\right.$ $.01)$ y el contenido para adultos $\left(\chi^{2}=43.25 ; p=.01\right)$ y actividades informativas como aplicaciones de radio por Internet $\left(\chi^{2}=18.60 ; p=.01\right)$.

Tabla 2

Comparación entre las actividades en Internet y grado de uso: controlado y problemático

\begin{tabular}{|c|c|c|c|c|c|}
\hline \multirow{2}{*}{ Actividades en Internet } & \multirow{2}{*}{ Aplicaciones } & Controlado & Problemático & \multirow{2}{*}{$\chi^{2}$} & \multirow{2}{*}{$P$} \\
\hline & & $n=371(\%)$ & $\mathrm{n}=\mathbf{4 0}(\%)$ & & \\
\hline \multirow{4}{*}{ Comunicación } & Correo & 83.5 & 87.5 & 11.61 & .04 \\
\hline & Redes sociales & 84 & 92.5 & 14.45 & .01 \\
\hline & Chat & 27.4 & 50 & 10.31 & .11 \\
\hline & Juegos online & 11.8 & 20 & 8.32 & .13 \\
\hline \multirow{3}{*}{ Información } & Académica & 54.4 & 87.5 & 3.12 & .68 \\
\hline & Radio por Internet & 25 & 52.5 & 18.60 & .01 \\
\hline & Periódicos & 40.7 & 42.5 & 4.88 & .43 \\
\hline \multirow{4}{*}{ Ocio } & Subasta & 4.5 & 15 & 14.81 & .01 \\
\hline & Video musicales & 69.8 & 82.5 & 7.52 & .18 \\
\hline & Contenido para adultos & 11.3 & 40 & 43.25 & .01 \\
\hline & Navegar en Internet & 72.7 & 82.5 & 16.07 & .41 \\
\hline
\end{tabular}

\section{Uso problemático de Internet y personalidad}

El análisis correlacional entre las puntuaciones directas del IAT y del BFI (tabla 3) evidenció: a) débil pero significativa correlación positiva con el factor neuroticismo; b) correlación negativa con los factores afabilidad y responsabilidad y; c) nula correlación con el factor extraversión y apertura a la experiencia. 
Tabla 3

Correlaciones entre las puntuaciones del IAT y del BFI

\begin{tabular}{|c|c|c|c|c|c|c|c|c|}
\hline & $M(p d)$ & $D T$ & 1 & 2 & 3 & 4 & 5 & 6 \\
\hline 1. IAT & 26.12 & 14.93 & 1 & & & & & \\
\hline 2. Extraversión & 26.02 & 5.67 & -.55 & 1 & & & & \\
\hline 3. Afabilidad & 31.42 & 5.46 & $-.25^{* *}$ & -.24 & 1 & & & \\
\hline 4. Responsabilidad & 1.00 & 5.29 & $-.31 * *$ & .37 & $.52^{* *}$ & 1 & & \\
\hline 5. Neuroticismo & 22.34 & 5.05 & $.20 * *$ & $-27^{* *}$ & -.31 & $-.16 * *$ & 1 & \\
\hline 6. Apertura & 33.61 & 6.38 & -.16 & .01 & $.55^{* *}$ & $.49 * *$ & $-.10 *$ & 1 \\
\hline
\end{tabular}

Pd: Puntaje directo; *Correlación significativa al .05 bilateral; ** Correlación significativa al 01 bilateral.

Con el propósito de identificar las variables predictoras del uso problemático de Internet se llevó a cabo una regresión logística binaria por pasos hacia adelante. La variable dependiente fue la categoría de uso, donde cero era uso controlado de Internet y uno uso problemático. Las covariables fueron las dimensiones de personalidad, el sexo y las actividades en Internet. Como se observa en la tabla 4, el modelo logró una clasificación correcta del $89.8 \%$ de los participantes. Los factores protectores de uso problemático fueron la dimensión de responsabilidad $(\mathrm{OR}=.879$; $\mathrm{IC}$ $95 \%=.816-.948)$ y el hecho de ser mujer $(\mathrm{OR}=3.863$ : IC $95 \%=1.822-8.188)$ y el factor de riesgo fue el neuroticismo $(\mathrm{OR}=1.150 ; \mathrm{IC} 95 \%=1.066-1.240)$. Las actividades de Internet y las dimensiones de personalidad extraversión, afabilidad y apertura no intervienen en el modelo final.

Tabla 4

Análisis de regresión logística binaria entre las categorías de uso de Internet, las dimensiones de personalidad y el sexo

\begin{tabular}{lcccccccc}
\hline \multicolumn{1}{c}{ Variables } & B & E.T & Walt & gl & p & OR & \multicolumn{1}{c}{ IC 95\% } \\
\hline Neuroticismo & 0.140 & .039 & 13.168 & 1 & .001 & 1.150 & 1.066 & 1.240 \\
Sexo & 1.351 & .383 & 12.428 & 1 & .001 & 3.863 & 1.822 & 8.188 \\
Responsabilidad & -0.129 & .038 & 11.315 & 1 & .001 & .879 & .816 & .948 \\
\hline
\end{tabular}

\section{Discusión}

Este es el primer estudio en América Latina que examina la asociación entre las dimensiones básicas de personalidad y el uso problemático de Internet en jóvenes, mediante el IAT (Puerta-Cortés et al., 2012) y el BFI (Benet-Martínez et al., 1988). Los resultados revelan que la mayoría de los estudiantes de la muestra $(90.2 \%)$ usaban Internet adecuadamente y desarrollaban actividades de comunicación, de búsqueda de información académica y de ocio. Internet es un espacio de acción individual y de expresión óptimo para los jóvenes porque se relaciona con la búsqueda de pertenencia, el desarrollo de la identidad y las necesidades de comunicación con sus pares y familiares (Aslanidou y Menexes, 2008; Gross, 2004).

El 9.7\% de la muestra presentó un uso problemático de Internet y no se encontró ningún caso de uso adictivo. La prevalencia obtenida en otros estudios que han utilizado el IAT es levemente superior: 10.2\% (Lam, Peng, Mai y Jing, 2009), 12.94\% (Liu et al., 2009) y 12\% (Puerta-Cortés et al., 2013). El grupo con uso problemático permanece más de cuatro horas diarias en Internet. Castellana et al. (2007) establecen que el adulto joven hace este tipo de uso cuando el número de horas de conexión afecta al correcto desarrollo de la vida cotidiana, causándole alteración del estado de ánimo y reducción de las horas dedicadas al estudio o a sus obligaciones. Puede darse también ansiedad o impaciencia por la lentitud de las conexiones o por no encontrar a quien busca, irritabilidad en el caso de interrupción y dificultad para salir de la pantalla.

El uso problemático de Internet se presentó más en hombres que en mujeres (Durke et al., 2012; Mottram y Fleming, 2009). Se relacionó con el desarrollo de actividades de comunicación como la revisión y el envío de correos electrónicos, el acceso a las redes sociales, las actividades de ocio como las subastas, las páginas de contenido para adultos y la actividad informativa como la radio. Los hombres con uso problemático de Internet desarrollan actividades de comunicación en la red para reducir los sentimientos de soledad (Çelik y Odaci, 2013) y la ansiedad social (Çuhadar, 2012). La participación en subastas y el acceso a paginas de contenido para adultos se relaciona con comportamientos compulsivos (Korkelia et al., 2010). El uso diferencial de los recursos de la red entre hombres y mujeres confirma el interés que despiertan las nuevas tecnologías entre los hombres (Muñoz-Rivas, Navarro y Ortega, 2003).

En cuanto a la relación entre el uso de Internet y la personalidad, nuestros resultados constatan, que el neuroticismo predice el uso problemático de Internet (Hamburger et al, 2000; Hardie et al, 2007; Viñas, 2009; Wolfradt et al., 2001). Los aspectos teóricos de la dimensión neuroticismo resal- 
tan el carácter descriptivo de los problemas psicológicos; las personas con puntuaciones altas en este factor tienden a experimentar emociones negativas (John et al., 1999). Internet es un medio para "sentir alivio" de los problemas cotidianos o las emociones displacenteras (García del Castillo et al., 2008; Muñoz-Rivas et al., 2003).

En la muestra estudiada, los jóvenes con uso problemático presentaron alto neuroticismo, baja afabilidad y baja responsabilidad. Para Kuss et al. (2013) las dimensiones de neuroticismo y afabilidad aumentarían la probabilidad de usar Internet de forma adictiva. La relación entre el uso problemático y la baja responsabilidad se debe posiblemente a dificultades en la organización, autodisciplina, cumplimiento de normas y compromisos (John et al., 1999). No se encontró relación entre apertura a la experiencia y el uso problemático de Internet, al igual que Landers et al. (2006). Sin embargo es necesario mencionar que esta dimensión presentó baja fiabilidad. Para Buckner et al. (2012), no hay relación clara entre el uso de Internet y apertura a la experiencia; además muchas de las variables de este factor no son fáciles de definir en términos de conducta (John et al., 1999).

El hecho de ser mujer y la alta responsabilidad son factores protectores; estas condiciones atenúan la posibilidad de uso adictivo de la red. Sin embargo, se requiere más investigación para fundamentar las relaciones encontradas. En investigaciones futuras sería necesario profundizar con el modelo dimensional de la personalidad y el uso de Internet, en especial apertura a la experiencia y extraversión, dimensiones que aún presentan resultados inconsistentes.

La metodología de cuestionarios online utilizada en este estudio reduce el sesgo de selección y la deseabilidad social de respuesta de los estudiantes y que los resultados sean más confiables. Para Ruiz-Olivares, Lucena, Pino y Herruzo (2010), los cuestionarios aplicados en el aula pueden estar sesgados porque los estudiantes que van a clase con regularidad son los que tienen, en general, hábitos de vida más saludables. Sin embargo, para mayor confiabilidad, de Gracia, Vigo, Fernández y Arbonés (2002), proponen contrastar los resultados con estudios realizados con metodologías tradicionales.

Este estudio presenta tres limitaciones a superar en futuras investigaciones. La primera se refiere a la fiabilidad de la versión adaptada a contextos de habla hispana del BFI de Benet-Martínez et al. (1988). Aunque la consistencia general del cuestionario obtenida es aceptable (0.73), la fiabilidad de las dimensiones no fue satisfactoria, en consonancia con los resultados de otros investigadores iberoamericanos con este instrumento (Bringas, Rodríguez y Herrero, 2009; de Brito et al., 2013; Puente y Cavazos, 2013). En consecuencia, se recomienda cautela en la utilización de éste instrumento. La segunda limitación se refiere a los participantes; aunque se trata de una muestra amplia, no es representativa y es necesario ser prudente en la generalización de los resultados, ya que solo se trata de estudiantes universitarios. La tercera limitación es inherente al diseño trasversal del estudio, sin embargo, la limitación de los estudios longitudinales es la supeditación a los rápidos cambios tecnológicos y a la evolución propia de la edad y las influencias del contexto.

Finalmente, a pesar de las limitaciones de este estudio exploratorio, se desprende que en el contexto latinoamericano, donde la penetración de Internet crece exponencialmente, es necesario analizar el uso adictivo de Internet en los jóvenes varones, para prevenir, intervenir y optimizar el uso de Internet.

\section{Reconocimientos}

Los autores agradecen a la Universidad de Ibagué la financiación del estudio referencia 11-220-COL0036482.

\section{Conflicto de intereses}

Los autores declaran que no tienen conflicto de intereses en este estudio.

\section{Referencias}

Amichai-Hamburger, Y. (2002). Internet and personality. Computers in Human Behavior, 18, 1-10. doi: 10.1016/j. chb.2010.03.018.

Aslanidou, S. y Menexes, G. (2008). Youth and the Internet: Uses and practices in the home. Computers $\mathcal{E}$ Education, 51, 1375-1391. doi: 10.1016/j.compedu.2007.12.003.

Benet-Martínez, V. y Jhon, O. (1988). Los cinco grandes across cultures and ethnic groups: Multitrait multimethod analyses of the Big Five in Spanish and English. Journal of Personality and Social Psychology, 75, 729-750.

Bringas, C; Rodríguez, F.J. y Herrero, F, J. (2009). Responsabilidad y comportamiento antisocial del adolescente como factores asociados al rendimiento escolar. Acta Colombiana de Psicología, 12 (2), 69-76.

Buckner, J. E., Castille, C. M. y Sheets, T. L. (2012). The five factor model of personality and employees excessive use of technology. Computers in Human Behavior, 28, 19471953. doi: 10.1016/j.chb.2012.05.014.

Caplan, S. E. (2003). Preference for online social interaction: A theory of problematic Internet use and psychosocial well-being. Communication Research, 30, 625-648. doi: $10.1177 / 0093650203257842$.

Carbonell, X., Chamarro, A., Beranuy, M., Griffiths, M., Oberst, U., Cladellas, R. y Talarn, A. (2012). Problematic Internet and cell phone use in Spanish teenagers and young students. Anales de Psicologia, 28, 789-796. doi: 10.6018/analesps.28.3.156061.

Castellana, M., Sánchez-Carbonell, X., Graner, C. y Beranuy, M. (2007). El adolescente ante las tecnologías de la in- 
formación y la comunicación: Internet, móvil y videojuegos. Papeles del Psicólogo, 28, 196-204.

Cao, F. y Su, L. (2007). Internet addiction among Chinese adolescents: Prevalence and psychological features. Child: Care, Health and Development, 33, 275-281.

Çelik, C. B. y Odaci, H. (2013). The relationship between problematic internet use and interpersonal cognitive distortions and life satisfaction in university students. Children and Youth Services Review, 35, 3, 505-508.

Costa, P. T., Jr. y McCrae, R. R (1985). The NEO personality inventory manual. Odessa, FL: Psychological Assessment Resources.

Çuhadar, C. (2012). Exploration of problematic Internet use and social interaction anxiety among Turkish pre-service teachers. Computers E्E Education, 59, 173-181.

de Brito, A. I, Veloso, V; da Silva, N; de Lima, M, de Oliveira, L. (2013). Escolha do (a) Parceiro (a) Ideal por Heterossexuais: São seus Valores e Traços de Personalidade uma Explicação? Psicologia: Reflexão e Crítica, 26, 29-37.

de Gracia, M., Vigo, M., Fernández, M. J. y Arbonés, M. (2002). Problemas conductuales relacionados con el uso de Internet: Un estudio exploratorio. Anales de Psicología, 18, 273-292.

Durkee, T., Kaess, M., Carli,V., Parzer, P., Wasserman, C., Floderus, B.,... Wasserman, D. (2012). Prevalence of pathological Internet use among adolescents in Europe: Demographic and social factors. Addiction, 107, 22102222. doi: 10.1111/j.1360-0443.2012.03946.x.

Echeburúa, E. y De Corral, (2010). Adicción a las nuevas tecnologías y a las redes sociales en jóvenes: un nuevo reto. Adicciones, 22, 91-96.

Engelberg, E. y Sjöberg, L. (2004). Internet use, social skills, and adjustment. CyberPsychology E Behavior, 7, 41- 47.

Estévez, L., Bayón, C., de la Cruz, J. y Fernández, A. (2009). Uso y abuso de Internet en adolescentes. En E. Echeburúa, F.J. Labrador y E. Becoña (eds.). Adicción a las nuevas tecnologías (101-130). Madrid: Pirámide.

Fossati, S., Borroni, S., Marchione, D. y Maffei, C. (2011). The Big Five Inventory (BFI). Reliability and validity of Italian translation in three independent nonclinical samples. European Journal of Psychological Assessment, 27, 50-58.

García del Castillo, J. A., Terol, M. C., Nieto, M., Lledó, A., Sánchez, S., Martín-Aragón, M. y Sitges, E. (2008). Uso y abuso de Internet en jóvenes universitarios. Adicciones, 20, 131-142.

Goldberg, L. R. (1993). The structure of phenotypic personality traits. American Psychologist, 48, 26-34. doi:10.1037/0003-066X.48.1.26.

Gross, E. (2004). Adolescent Internet use: What we expect, what teens report. Applied Developmental Psychology, 25, 633-649. doi:10.1016/j.appdev.2004.09.005.

Hamburger, Y. A. y Ben-Artzi, E. (2000). The relationship between extraversion and neuroticism and the different uses of the Internet. Computers in Human Behavior, 16, 441- 449. doi:10.1016/S0747-5632 (00).

Hardie, E. y Tee, M. (2007). Excessive Internet use: The role of personality, loneliness and social support networks in Internet addiction. Australian Journal of Emerging Technologies and Society, 5, 34-47.

John, O. P., Donahue, E. M. y Kentle, R. L. (1991). The Big Five Inventory-Versions $4 a$ and 54. Berkeley, CA: University of California, Berkeley, Institute of Personality and Social Research.

John, O. P. y Srivastava, S. (1999). The big five trait taxonomy: History, measurement, and theoretical perspectives. University of California at Berkeley. In L. Pervin and O.P. John (Eds.), Handbook of personality: Theory and research (2nd ed.). New York: Guilford.

Kim, K., Ryu, E., Chon, M. Y., Yeun, E. J., Choi, S. Y., Seo, J. S. y Nam, B. W. (2006). Internet addiction in Korean adolescents and its relation to depression and suicidal ideation: a questionnaire survey. International Journal of Nursing Studies, 43, 185-192.

Korkelia, J., Kaarlas, S., Jääskeläinen, M., Vahlberg, T. y Taiminen, T. (2010). Attached to the web harmful use of the Internet and its correlates. European Psychiatry, 25, 236-241.

Kuss, D. J., Griffiths, M. D. y Binder, J. F. (2013). Internet addiction in students: Prevalence and risk factors. Computers in Human Behavior, 29, 959-966.

Lam, L., Peng, Z., Mai, J. y Jing, J. (2009). Factors associated with Internet addiction among adolescents. CyberPsychology Eo Behavior, 12(5), 551-555.

Landers, R. N. y Lounsbury, J. W. (2006). An investigation of Big Five and narrow personality traits in relation to Internet usage. Computers in Human Behavior, 22, 283293.

Liu, Q., Xiao, S., Cao, H., Xu, H., Zhou, L. y Luo, D. (2009). Relationship between personality characteristics and quality of life in college students with different extent of Internet use. Chinese Mental Health Journal, 23, 138-142.

Luo, T. y Ding, D. (2006). Relationships among personality traits, motive of Internet use, and tendency of Internet addiction. Chinese Journal of Clinical Psychology, 14, 365367.

McCrae, R. R. y Costa, P. T. (1997). Personality trait structure as a human universal. American Psychologist, 52, 509516.

McCrae, R. R. y Costa, P. T. (2008). The five-factor theory of personality. En O. P. John, R. W. Robins and L. A. Pervin (Eds.), Handbook of personality: Theory and research (3rd ed., pp. 182-207). New York: Guilford Press.

Montag, C., Jurkiewicz, M. y Reuter, M. (2010). Low self-directedness is a better predictor for problematic Internet use than high neuroticism. Computers in Human Behavior, 26, 1531-1535. 
Moreno, M., Jelenchick, L., Cox, E., Young, H. y Christakis, D. (2011). Problematic Internet use among US youth: A systematic review. Archives of Pediatrics and Adolescent Medicine, 165, 797-805. doi:10.1001/archpediatrics.2011.58.

Mottram, A. J. y Fleming, M. J. (2009). Extraversion, impulsivity, and online group membership as predictors of problematic Internet use. CyberPsychology E Behavior, 12, 319-321. doi: 10.1089/cpb.2007.0170.

Muñoz-Rivas, M. J., Navarro, M. E. y Ortega, N. (2003). Patrones de uso de Internet en población universitaria española. Adicciones, 15, 137-144.

Puente, R. y Cavazos, J. (2013). Personality factors, affect, and autonomy support as predictors of life satisfaction. Universitas Psychologica, 12, 41-54.

Puerta-Cortés, D. X. y Carbonell, X. (2013). Uso problemático de Internet en una muestra de estudiantes universitarios colombianos. Avances en Psicología Latinoamericana, 31, 620-631.

Puerta-Cortés, D. X, Carbonell, X. y Chamarro, A. (2012). Análisis de las propiedades psicométricas de la versión en español del Internet Addiction Test. Trastornos Adictivos, 14, 99-104.

Ruiz-Olivares, R., Lucena, V., Pino, J. y Herruzo, J. (2010). Análisis de comportamientos relacionados con el uso/ abuso de Internet, teléfono móvil, compras y juego en estudiantes universitarios. Adicciones, 22, 301-310.

Sánchez-Carbonell, X., Beranuy, M., Castellana, M., Chamarro, A. y Oberst, U. (2008). La adicción a Internet y al móvil: ¿moda o trastorno? Adicciones, 20, 149-160.

Thalmayer, A. G., Saucier, G. y Eigenhuis, A. (2011). Comparative validity of brief to medium-length Big Five and Big Six. Personality Questionnaires Psychological Assessment, 23, 995-1009. doi: 10.1037/a0024165.

Triandis, H. C. y Eunkook, M. S. (2002). Cultural influences on personality. Annual Review of Psychology, 53, 133160 .

Tendencias Digitales (2012). Iconografía usos de Internet en Latinoamérica. Recuperado de http://www.tendenciasdigitales.com/
Tsai, H. F., Cheng, S. H., Yeh, T. L., Shih, C. C., Chen, K. C., Yang, Y. C. y Yang, Y.K.(2009). The risk factors of Internet addiction: A survey of university freshmen. Psychiatry Research, 167, 294-299. doi: 10.1016/j.psychres.2008.01.015.

van den Eijnden, R., Spijkerman , R., Vermulst, A., van Rooij, T. y Engels, R.(2009). Compulsive Internet use among adolescents: Bidirectional parent-child relationships. Journal of Abnormal child Psychology, 38, 77-89. doi:10.1007/s10802-009-9347-8.

Viñas, F. (2009). Self-reported use of Internet among adolescents: Psychological profile of elevated Internet use. International Journal of Psychology and Psychological Therapy, 9, 109-122.

Witt, E. A., Massman, A. J. y Jackson, L. A. (2011). Trends in youth's videogame playing, overall computer use, and communication technology use: The impact of self-esteem and the Big Five personality factors. Computers in Human Behavior, 27, 763-769. doi: 10.1016/j. chb.2010.10.025.

Wolfradt, U. y Doll, J. (2001). Motives of adolescents to use the Internet as a function of personality traits, personal and social factors. Journal of Educational Computing Research, 24, 13-27. doi: 10.2190/ANPM-LN97-AU97AUT2-D2EJ.

Young, K. S. (1998). Caught in the Net: How to recognize the signs of Internet addiction and a winning strategy for recovery. New York: John Wiley \& Sons, Inc.

Young, K. S. (2004). Internet Addiction. A new clinical phenomenon and its consequences. American Behavioral Scientist, 48, 402-415. doi: 10.1177/0002764204270278.

Yellowlees, P. M. y Marcas, S. (2007). Problematic Internet use or Internet addiction? Computers in Human Behavior, 23, 1447-1453. doi: 0.3109/00952990.2010.491880. 